\title{
Green ultrasound-assisted extraction of bioactive compounds from button mushrooms, potato and onion peels
}

Elenilson G. Alves Filho ${ }^{a *}$; Marcelo Lima ${ }^{\text {; }}$ Lorena Silvac; Paulo Ribeiroc; Brijesh K. Tiwarid; Fabiano N. Fernandes ${ }^{\mathrm{b}}$; Edy S. Brito ${ }^{\mathrm{c} *}$

a Universidade Federal do Ceará, Departamento de Engenharia de Alimentos, Campus do Pici, Bloco 858, 60440-900 Fortaleza, CE, Brazil.

b Universidade Federal do Ceará, Departamento de Engenharia Química, Campus do Pici, Bloco 709, 60440-900 Fortaleza, CE, Brazil.

` Embrapa Agroindústria Tropical, 60511-110 Fortaleza, CE, Brazil.

d Teagasc, Ashtown Food Research Centre, Dublin 15, D15 DY05, Ireland

* Corresponding authors:

E-mail address:

elenilson.godoy@yahoo.com.br (E.G. Alves Filho)

edy.brito@embrapa.br (E.S. de Brito) 
Figures S1, S3 and S5 illustrate the chromatograms (on same intensities) acquired under the negative ionization mode of the extracts from potato peels, onion peels, and mushrooms respectively, subjected to ultrasound processing followed by agitation. Figures S2, S4 and S6 present the semi-quantitative results of the potato peels, onion peels, and mushrooms respectively.

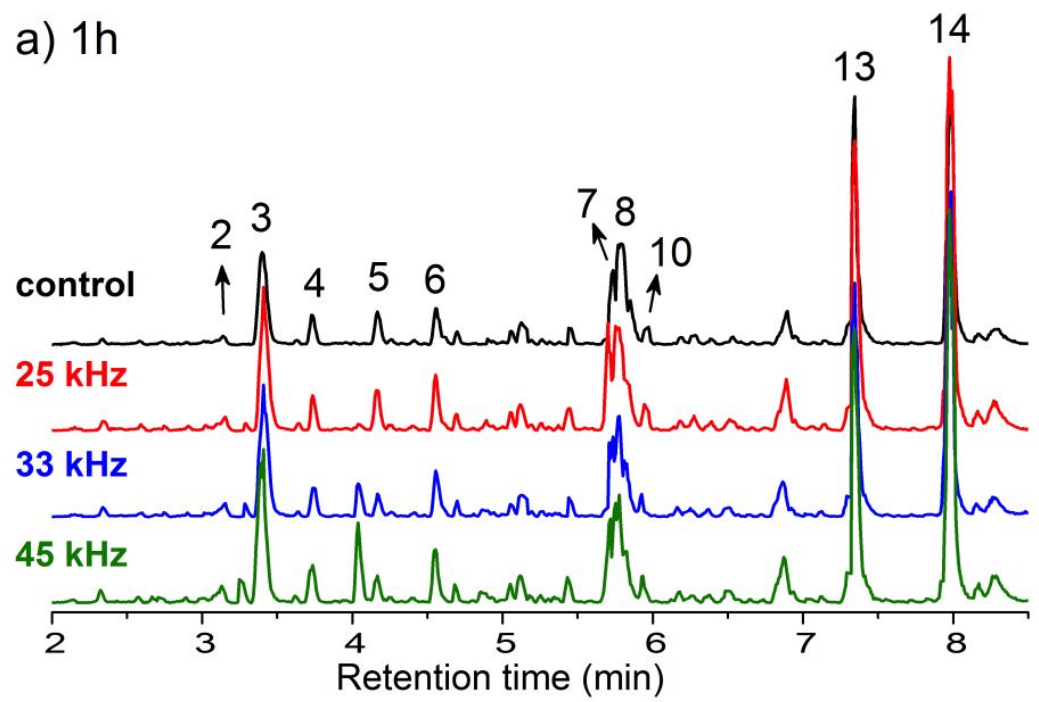

b) $16 \mathrm{~h}$

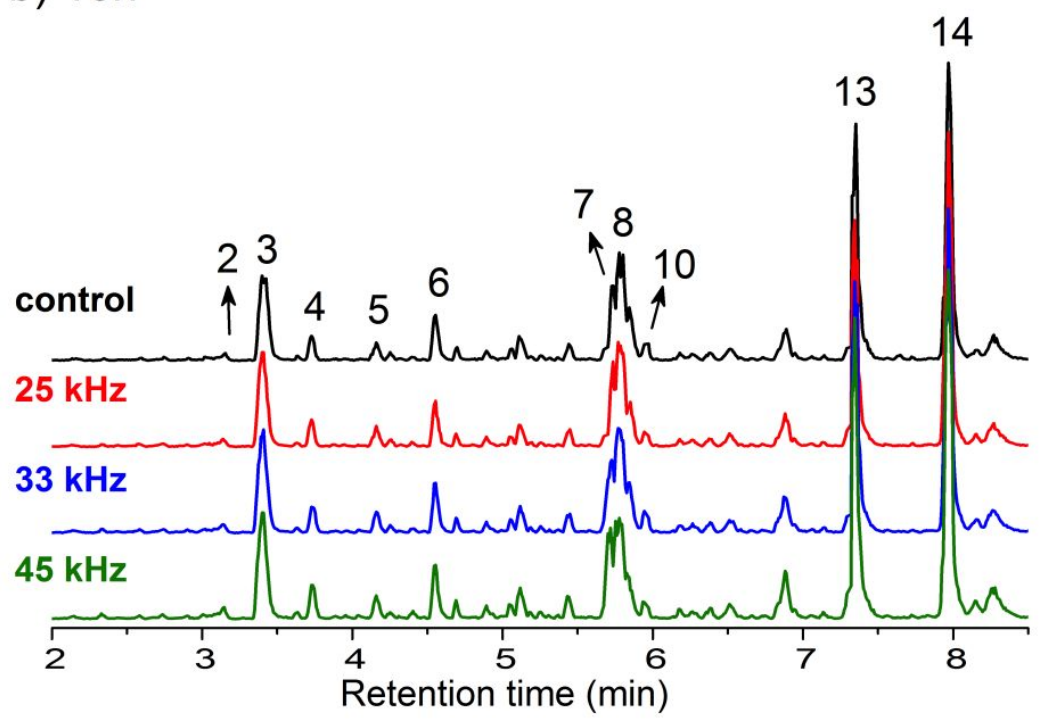

Figure S1. Chromatograms from potato peel extracts after ultrasound processing at 25, 33, and $45 \mathrm{kHz}$, followed by agitation during $1 \mathrm{~h}$ (a) and $16 \mathrm{~h}$ (b). The compounds numbers refer to Table 1: piscidic acid (2); non-identified (3); solsodomine A (4); 
kumatakenin (5); cis-feruloyl-octopamine (6); $\alpha$-solanine (7); $\beta$-chaconine (8); $\beta$ chaconine isomer (10); and 3-hydroxyoctanoic acid isomers (13 and 14).

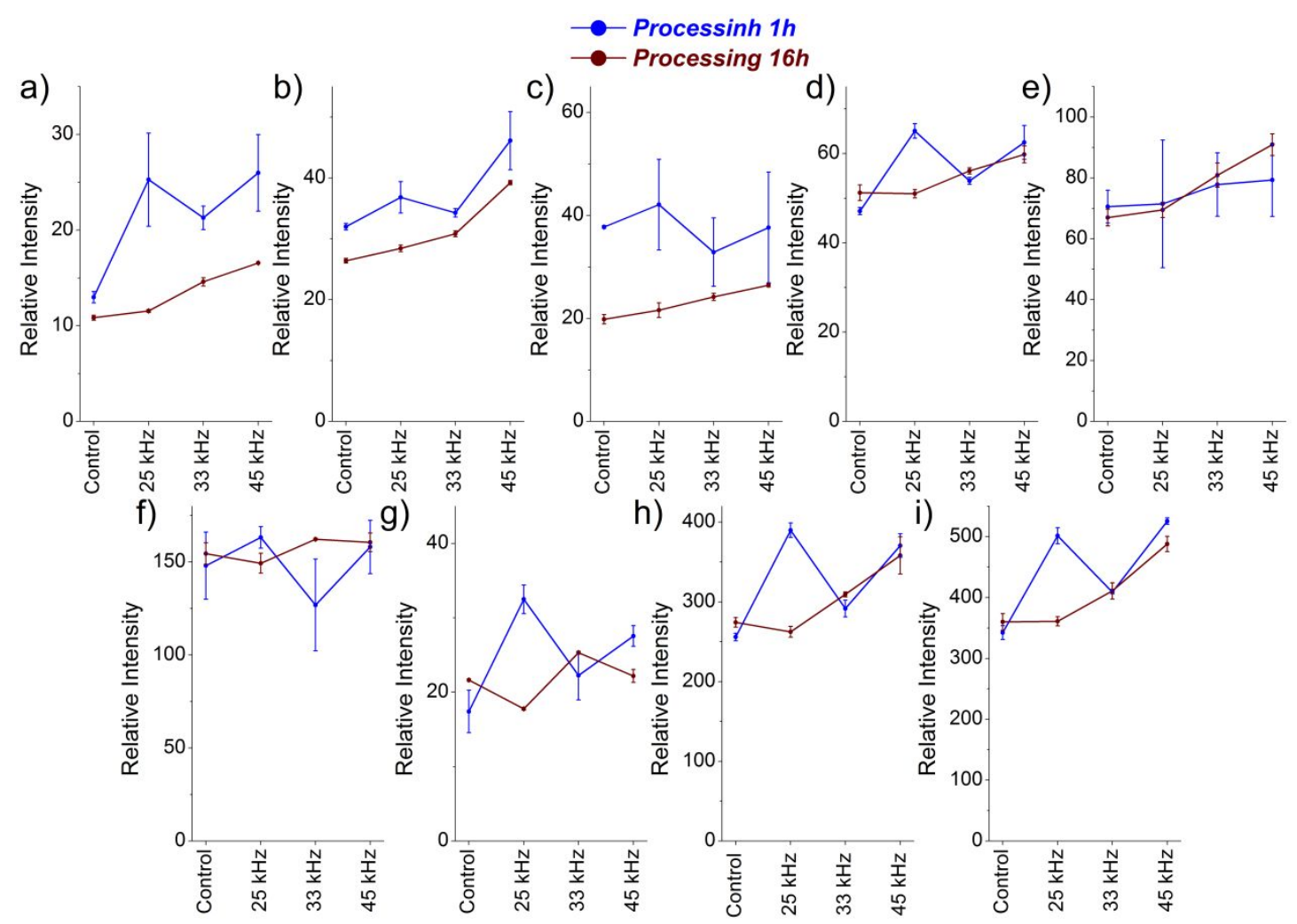

Figure S2. Quantitative results from the ANOVA single factor of the detected compounds in potato peel extracts after $1 \mathrm{~h}$ (blue) and $16 \mathrm{~h}$ (red) of processing: piscidic acid (a), solsodomine A (b), kumatekenin (c), cis-feruloylocropamine (d), $\alpha$ solanine (e), $\beta$-chaconine (f), $\beta$-chaconine isomer $(\mathrm{g})$, and trihydroxyoctanoic acid isomer (h and i). 


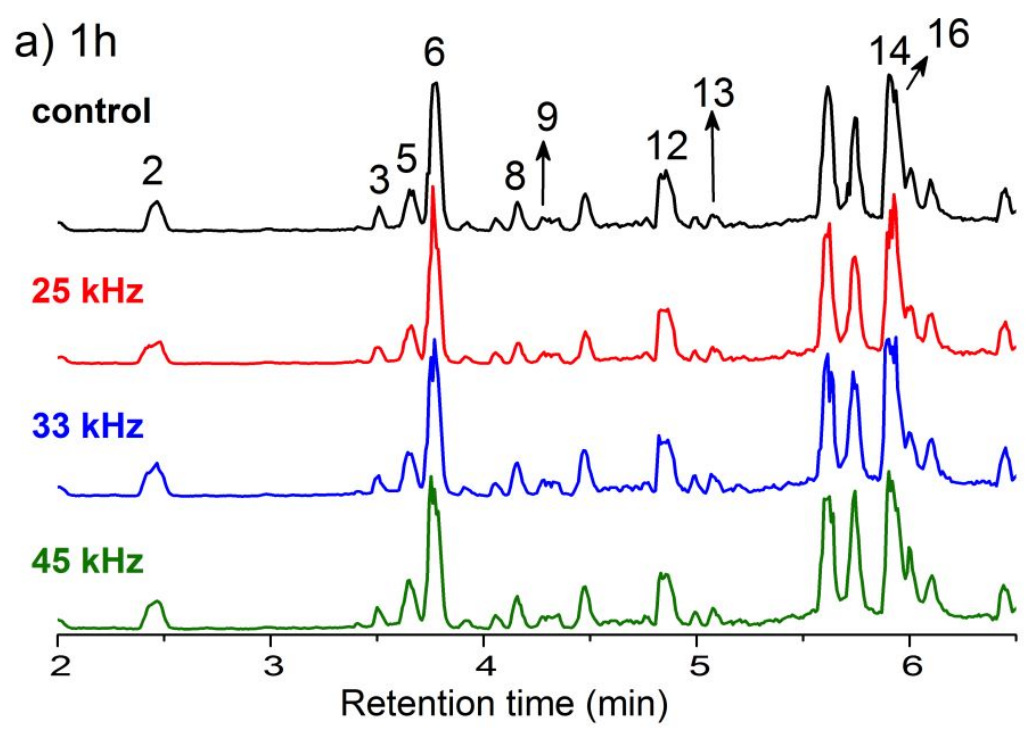

b) $16 \mathrm{~h}$

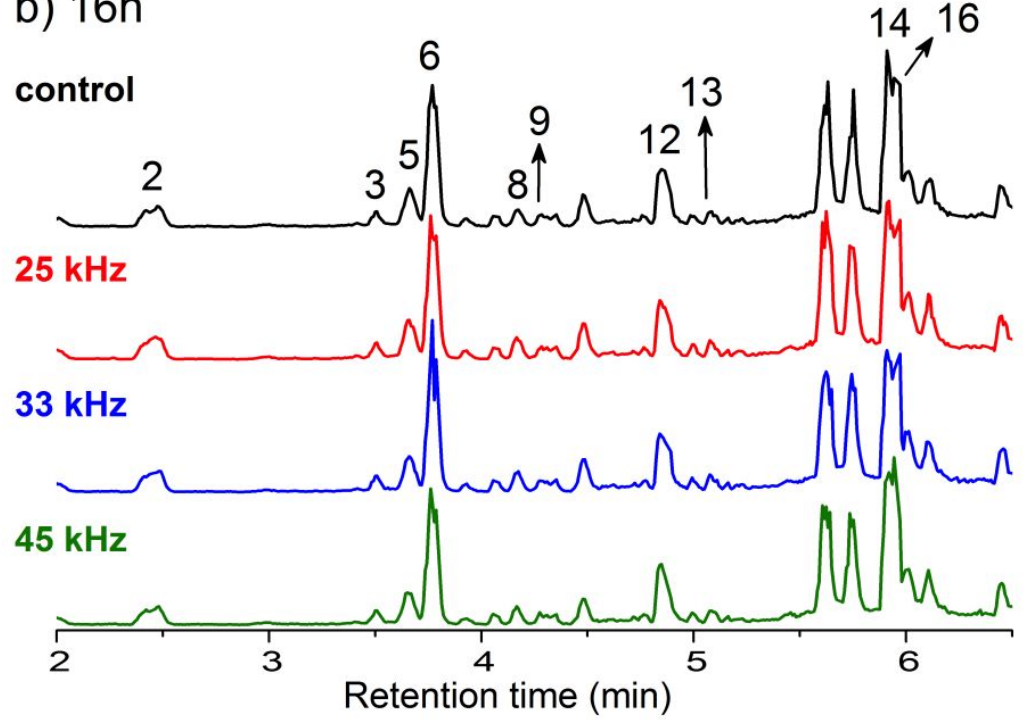

Figure S3. Chromatograms from the onion peels extracts after ultrasound processing at 25,33 , and $45 \mathrm{kHz}$ followed by agitation for $1 \mathrm{~h}$ (a) and $16 \mathrm{~h}$ (b). The compounds numbers are referred to Table 2: protocatechuic acid (2); quercetin-3,4`-diglucoside (3); myricetin (5); isorhamnetin-3,4`-diglucoside (6); ferulic acid (8); vanillic acid (9); quercetin-4`-glucoside (12); isorhamnetin-4 '-glucoside (13); quercetin (14); and quercetin isomer (16). 


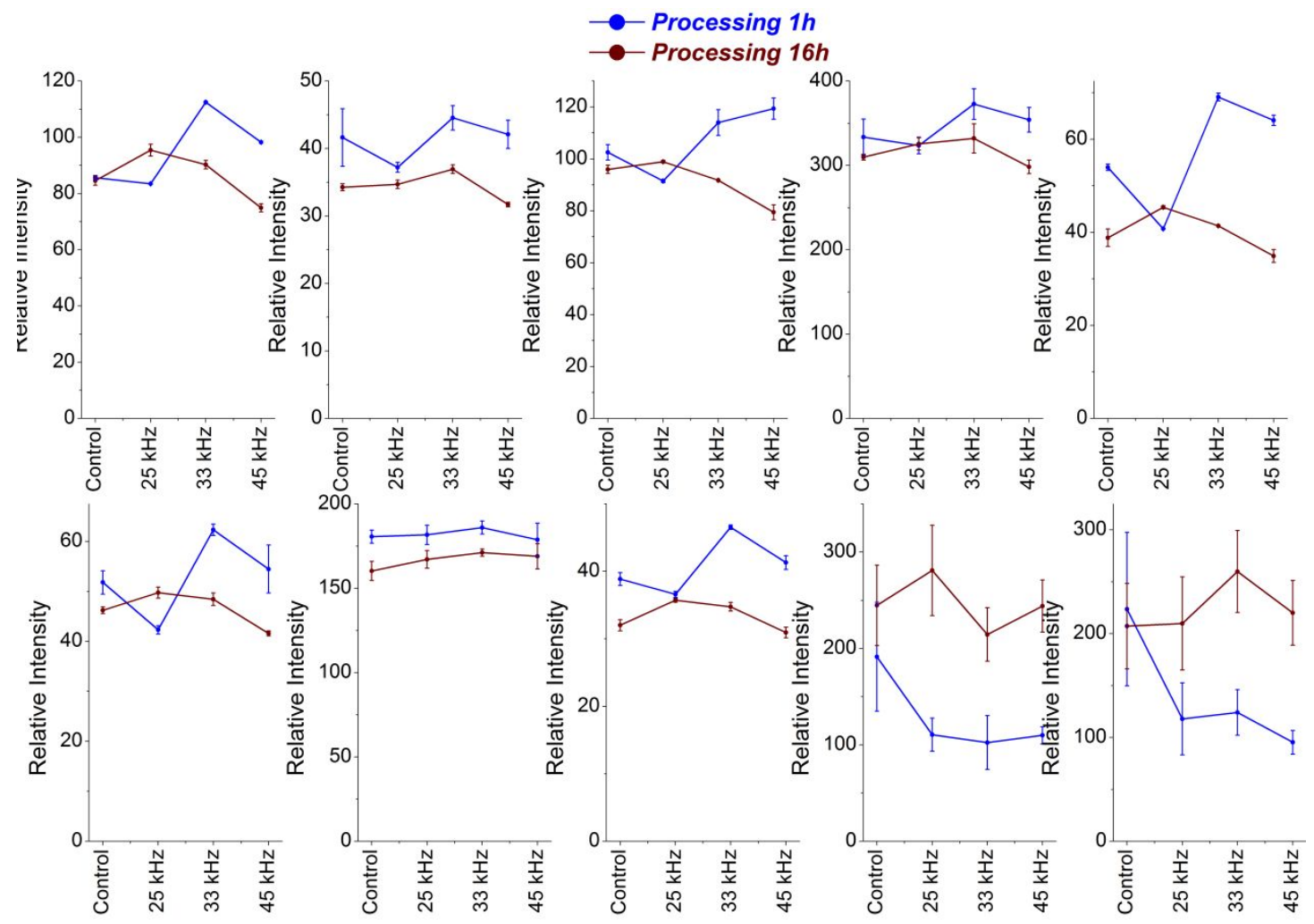

Figure S4. Quantitative results from the ANOVA single factor of the detected compounds in onion peels extracts. The compounds numbers refer to Table 2: protocatechuic acid (a); quercetin-3,4`-diglucoside (b); myricetin (c); isorhamnetin3,4`-diglucoside (d); ferulic acid (e); vanilic acid (f); quercetin-4`-glucoside (g); isorhamnetin-4 -glucoside (h); quercetin (i); and quercetin isomer (j). 
a) $1 \mathrm{~h}$

control
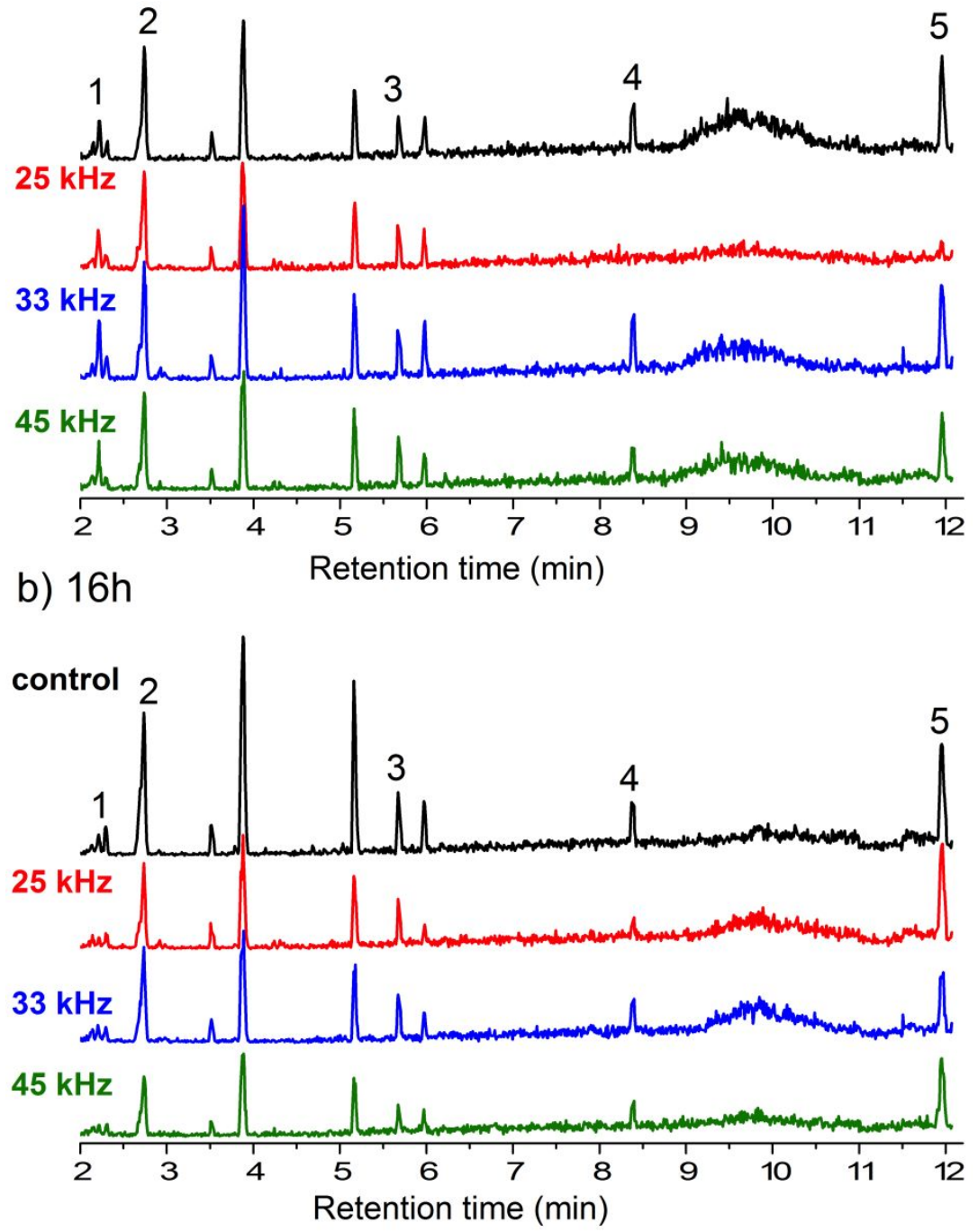

Figure S5. Chromatograms from mushroom extracts after ultrasound processing at 25,33 , and $45 \mathrm{kHz}$ of frequency followed by agitation for $1 \mathrm{~h} \mathrm{(a)}$ and $16 \mathrm{~h} \mathrm{(b).} \mathrm{The}$ compounds numbers refer to Table 3: succinyladenosine (1); tryptophan (2); 3(adenin-9-yl)-(RS)-alanine (3); 5(S),8(R)-dihydroxy monocarboxylic acid (4); and lysophosphatidylethanolamine 16:0 (5). 


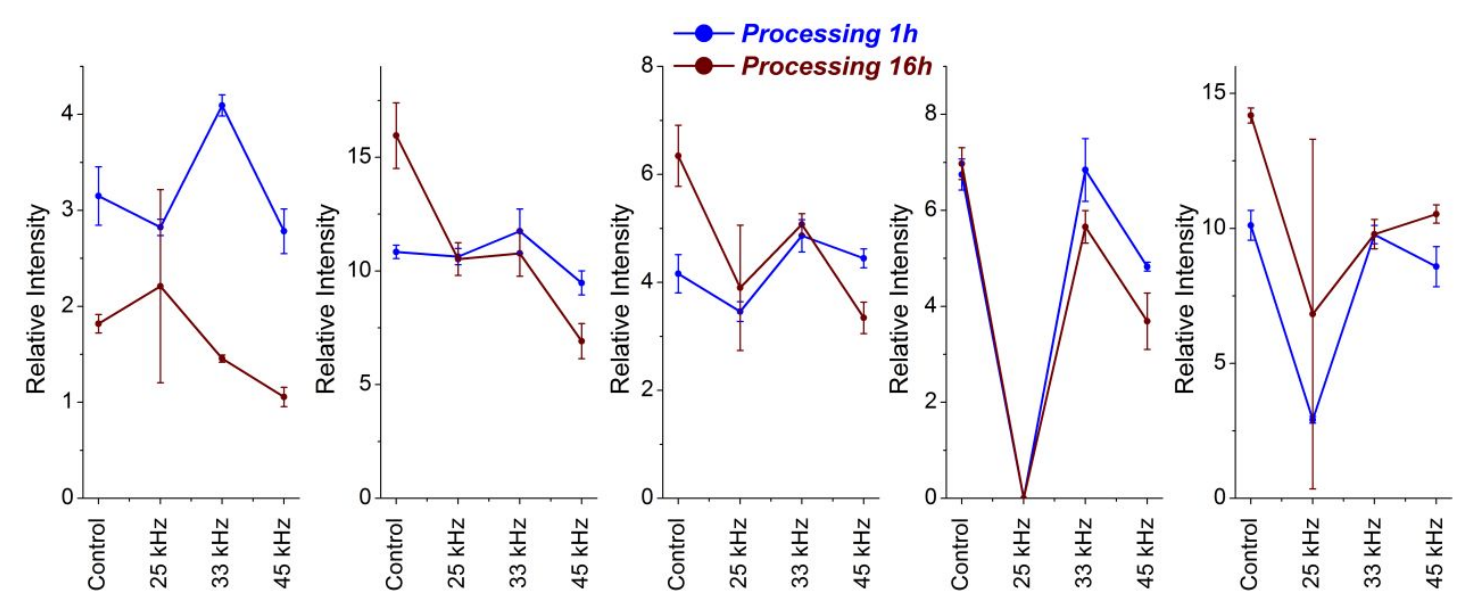

Figure S6. Quantitative results from the ANOVA single factor of the detected compounds in mushroom extracts after $10 \mathrm{~min}$ of sonication followed by $1 \mathrm{~h}$ (blue) and $16 \mathrm{~h}$ (red) of agitation. The compounds numbers refer to Table 3: succinyladenosine (a); tryptophan (b); 3-(adenin-9-yl)-(RS)-alanine (c); 5(S),8(R)dihydroxy monocarboxylic acid (d); and lysophosphatidylethanolamine 16:0 (e). 